\title{
Spherically Symmetric Dynamical Horizons
}

\author{
Robert Bartnik* and James Isenberg ${ }^{\dagger}$
}

November 22, 2018

\begin{abstract}
We study spherically symmetric dynamical horizons (SSDH) in spherically symmetric Einstein/matter spacetimes. We first determine sufficient and necessary conditions for an initial data set for the gravitational and matter fields to satisfy the dynamical horizon condition in the spacetime development. The constraint equations reduce to a single second order linear "master" equation, which leads to a systematic construction of all SSDH initial data sets with certain boundedness conditions. Turning from construction to existence, we find necessary and sufficient conditions for a given spherically symmetric spacetime to contain a SSDH.
\end{abstract}

2000 Mathematics Subject Classification: 53C99,83C57.

Keywords and Phrases: Einstein equations, dynamical horizon, black hole

\section{Introduction}

Since their introduction four years ago by Ashtekar and his collaborators [3] 4, dynamical horizons have been found to be very useful for the study of the dynamical formation of black holes. Among other things, they have been used to study gravitational wave fluxes and their influence on the areas of black holes as they develop, and they have been used in building analogues

${ }^{*}$ School of Mathematical Sciences, Monash University, Victoria, 3800 Australia. E-mail: robert.bartnik@sci.monash.edu.au

${ }^{\dagger}$ Department of Mathematics and Institute for Theoretical Science, University of Oregon, Eugene, OR 97403 USA. E-mail:jim@newton.uoregon.edu 
of black hole thermodynamics for astrophysical systems evolving into black holes [4].

How extensively do dynamical horizons occur in solutions of Einstein's equations? While there have been studies, both numerical and analytical, of certain families of explicit examples [6], no attempt has yet been made to determine the parameter space of solutions which admit them. We do this here for the special case of spherically symmetric solutions.

We obtain two types of results, on the construction of spacetimes with dynamical horizons (by constructing dynamical horizon initial data), and on locating dynamical horizons in given spacetimes.

We first consider the problem of constructing initial data satisfying both the dynamical horizon condition, and the Einstein constraint equations. Proposition 2 parametrizes those initial data on $I \times S^{2}$ which are spherically symmetric, which solve the Einstein constraint equations, and which serve as a dynamical horizon for their spacetime development. We find that the collection $\mathcal{D}$ of such initial data is parametrized by two free functions $(\xi, \tau)$ on an interval and a pair of real constants $\left(y_{0}, y_{1}\right)$. That is, for every choice of these two functions, we can generate an explicit initial data set of this sort; conversely, every data set in $\mathcal{D}$ can be generated in this way. For simplicity we consider only bounded potentials, but there are many examples of dynamical horizons with unbounded potentials [3, 6], whose global properties can be studied using similar techniques.

Note that since spherically symmetric solutions of the vacuum Einstein equations contain no dynamical horizons ${ }^{1}$, we consider here the Einstein constraint equations with generic matter, represented by an energy density field $\rho$ and a radial momentum density field $\xi$.

Second, we consider the problem of finding dynamical horizon(s) in a given (smooth, globally hyperbolic) spacetime. Lemma 7 and Proposition 8 give conditions on the stress-energy tensor which lead naturally to a general existence result for dynamical horizons in the spacetime.

Our arguments depend crucially on two quite interesting and novel identities: a constraint (8) on two components of the stress-energy tensor, and a reformulation of the spherically symmetric constraint equations as a linear second order ordinary differential equation (9) for the volume function $y=r^{3}$. We expect these expressions will play an important role in extending

\footnotetext{
${ }^{1}$ By Birkhoff's theorem the vacuum region is locally Schwarzschild, which has vanishing outer expansion only on the horizons, which are not spacelike.
} 
our results to non-spherical spacetimes.

\section{Preliminaries}

We work here with four dimensional spacetimes $(M, g, T)$ satisfying the Einstein gravitational field equations $G_{\mu \nu}=8 \pi \mathrm{G} T_{\mu \nu}$, where $M$ is a spacetime manifold, $g$ is a Lorentz signature metric invariant under an $S O(3)$ action and with Einstein curvature tensor $G_{\mu \nu}=R_{\mu \nu}-\frac{1}{2} R g_{\mu \nu}, T_{\mu \nu}$ is a stress-energy tensor field which may or may not be a functional of a specific set of field variables, and $\mathrm{G}$ is the Newton gravitational constant. In our spacetime results (Section 4) we require that $(M, g, T)$ satisfy an energy condition; in our initial data results (Section 3), such a condition is not needed.

Definition 1 An embedded hypersurface $\Sigma^{3}$ in a spacetime $(M, g, T)$, is a dynamical horizon for the spacetime if the following conditions are met:

(DH1) $\Sigma^{3}$ is spacelike;

(DH2) $\Sigma^{3}$ is foliated by marginally outer-trapped 2-surfaces, i.e., surfaces such that the expansion of the outer future null vector field $\ell=\ell_{+}$vanishes, $\theta_{\ell}=\theta_{+}=0$

(DH3) the marginally outer trapped 2-surfaces are inner trapped, $\theta_{n}=\theta_{-}<0$, where $n=\ell_{-}$is the inward future null vector field normat ${ }^{2}$ to the 2surfaces.

A 2-surface with null normal expansions satisfying (DH2) and (DH3) is said to be marginally trapped. A hypersurface satisfying just (DH2) and (DH3) is called a marginally trapped tube [2] however here we are concerned only with the dynamical horizon case. Under spherical symmetry we may assume the 2-surfaces are isometric 2-spheres of positive radius.

A spacetime may generally contain a number of dynamical horizons. However, as shown in [2], the foliation of a given dynamical horizon by marginally trapped surfaces is unique. It also follows from the results in 2 that if a given spacetime satisfies the null energy condition (i.e., $T(\ell, \ell) \geq 0$ for every null vector $\ell$ ), then no spacetime region admits a local foliation by dynamical horizons.

\footnotetext{
${ }^{2}$ normalised by $g(n, \ell)=-2$.
} 


\section{Constructing Initial Data}

In this section, we derive an algorithm for the construction of spherically symmetric initial data sets which satisfy both the constraint equations and the dynamical horizon condition. In doing so we determine the freely specifiable parts of such initial data, showing that there is a bijective correspondence between this free data on the one hand, and spherically symmetric dynamical horizon solutions of the constraints on the other hand. We then describe some example solutions, and we discuss some physically motivated restrictions one might impose on these solutions.

\subsection{Construction Algorithm and the Free Data}

An initial data set for the Einstein equations consists of $\left(\Sigma^{3}, \gamma, K, \rho, J\right)$, where $\Sigma^{3}$ is a three dimensional manifold (the initial slice), $\gamma$ is a Riemannian metric (the initial metric), $K$ is a symmetric tensor (the initial extrinsic curvature), $\rho$ is a non-negative function ( $8 \pi \mathrm{G}$ times the initial matter energy density), and $J$ is a one form field ( $8 \pi \mathrm{G}$ times the initial momentum density). Assuming this data satisfies the Einstein constraint equations,

$$
\begin{aligned}
2 \rho & =R_{\gamma}-K_{a b} K^{a b}+\left(\operatorname{tr}_{\gamma} K\right)^{2} \\
J_{a} & =\nabla^{b} K_{a b}-\nabla_{a}\left(\operatorname{tr}_{\gamma} K\right)
\end{aligned}
$$

where $R_{\gamma}$ is the scalar curvature of $\gamma$, it follows (assuming appropriate matter evolution equations) [10] [1] [7] that there is a unique spacetime $\left(\Sigma^{3} \times I, g, T\right)$ which i) satisfies the Einstein gravitational and matter field equations, ii) induces the initial data $\left(\Sigma^{3}, \gamma, K, \rho, J\right)$ on the embedded hypersurface ${ }^{3} \Sigma^{3} \times$ $\{0\}$, iii) is globally hyperbolic, and iv) contains (up to diffeomorphism) all other spacetimes which satisfy conditions i)-iii). This spacetime is called the maximal spacetime development of $\left(\Sigma^{3}, \gamma, K, \rho, J\right)$. These results allow us to focus on the simpler initial data constraint system, rather than confront the challenges of the full evolution equations. Note, however, that if we are given a pair of solutions of the constraint equations, it is generally not easy to determine whether or not their spacetime developments are diffeomorphic.

\footnotetext{
${ }^{3}$ in the sense that $\gamma$ is the pullback of $g$ to $\Sigma^{3} \times\{0\}, K$ is the second fundamental form corresponding to $\Sigma^{3} \times\{0\}, \rho=8 \pi \mathrm{G} T\left(e_{\perp}, e_{\perp}\right)$ for $e_{\perp}$ the future-pointing timelike normal to $\Sigma^{3} \times\{0\}$, and $J=8 \pi \mathrm{G} T\left(e_{\perp}, \cdot\right)$
} 
If we restrict our attention to initial data sets which are spherically symmetric, with $\Sigma^{3}$ diffeomorphic to the three dimensional annulus $I \times S^{2}$, then we may write

$$
\begin{aligned}
\gamma & =d R^{2}+r^{2}(R)\left(d \theta^{2}+\sin ^{2} \theta d \phi^{2}\right), \\
K & =\frac{1}{2} \mu(R) \gamma+\left(\tau(R)-\frac{3}{2} \mu(R)\right) d R^{2},
\end{aligned}
$$

and $J=\xi(R) d R$, where $R \in\left(R_{0}, R_{1}\right), \theta \in(0, \pi)$ and $\phi \in(0,2 \pi)$ ). (Note that the coordinate $R$ measures radial geodesic distance, and $r(R)$ is the spherical radius function.) Hence the full set of (spherically symmetric) initial data is parametrized by five real valued functions: $r(R), \mu(R), \tau(R), \rho(R)$ and $\xi(R)$. Note that $\tau=\operatorname{tr}_{\gamma} K$ is the mean curvature of the spacetime hypersurface $\Sigma$, whilst $\mu$ is the mean curvature in $\Sigma$ of the 2 -spheres $S_{r}^{2}$ of constant radius $r$.

The constraint equations (12) for spherical data take the form

$$
\begin{aligned}
2 r \frac{d^{2} r}{d R^{2}} & =-\left(\frac{d r}{d R}\right)^{2}+1-r^{2}\left(\frac{3}{4} \mu^{2}-\mu \tau+\rho\right), \\
r \frac{d \mu}{d R} & =(2 \tau-3 \mu) \frac{d r}{d R}-r \xi .
\end{aligned}
$$

We wish to study spherically symmetric solutions of the constraint equations which serve as dynamical horizons for their spacetime developments. The dynamical horizon condition $\theta_{+}=0$ takes the form

$$
\theta_{\ell}=\theta_{+}=\mu+\frac{2}{r} \frac{d r}{d R}=0,
$$

where $\mu$ is the trace of the extrinsic curvature $K$ over the $S^{2}$ tangent 2-planes. Substituting for $\mu$ in (15) and (6) gives two equations for $\frac{d^{2} r}{d R^{2}}$, and eliminating this common term gives

$$
\rho+\xi=\frac{1}{r^{2}} .
$$

We thus obtain a somewhat peculiar, quite explicit, condition on the matter field part of the initial data which must be satisfied if the data set is to correspond to a dynamical horizon.

Before stating the main result on the existence and parameterization of initial data, we note another curious property: substituting (8) and (7) in (5) leads to a linear "master" equation for $y:=r(R)^{3}$ :

$$
\frac{d^{2} y}{d R^{2}}+\tau \frac{d y}{d R}-\frac{3}{2} \xi y=0 .
$$


Proposition 2 Suppose we are given functions $\tau, \xi \in C^{0}(\mathbb{R})$ and a solution $y=y(R)$ of (9) on an interval $I=\left(R_{1}, R_{2}\right)$ on which $y$ is everywhere positive. Defining $r=y^{1 / 3}, \mu$ by (7) and $\rho$ by (8) gives a solution of the spherical constraint equations (3 6). Conversely, (8,9) hold if the given data $(r, \mu, \tau, \rho, \xi)$ satisfy (3 6) and (7) on an interval where $r$ is everywhere positive.

Furthermore, let $y=y(R)$ be the solution of (9) with initial conditions

$$
y\left(R_{0}\right)=y_{0}>0, \quad \frac{d}{d R} y\left(R_{0}\right)=y_{1}>0,
$$

and denote by $I=\left(R_{1}, R_{2}\right), R_{0} \in I$, the maximal interval on which the condition $r>0$ holds. Let $(y, r, \mu, \tau, \rho, \xi)$ be the corresponding solution of (39). If $R_{1}>-\infty$ then we may normalise $R$ so $R_{1}=0$ and then $\lim _{R \downarrow 0} r(R)=$ 0 and $\gamma$ is singular at $R_{1}$; likewise if $R_{2}<\infty$ then $\lim _{R \uparrow R_{2}} r(R)=0$ and $\gamma$ is singular at $R_{2}$.

The solution $(y, r, \mu, \tau, \rho, \xi)$ on the interval $\left(R_{1}, R_{2}\right)$ specifies a dynamical horizon if additionally $\frac{d}{d R} y>0$ on $\left(R_{1}, R_{2}\right)$.

Proof: Direct computation verifies the equivalence of the spherically symmetric constraint equations (3) 6) to the linear equation (91), augmented by formulas (7) for $\mu$, and (8) for $\rho$. This proves the first part of the proposition.

The continuity assumptions $\tau, \xi \in C^{0}(\mathbb{R})$ ensure that the initial value problem for the linear ODE (9) together with the the initial conditions (10) has a unique global solution $y \in C^{2}(\mathbb{R})$ which is positive in a neighborhood of $R_{0}$; hence $\lim _{R \downarrow R_{1}} y(R)$ exists and the solution $y(R)$ extends to $R<R_{1}$. By maximality of $I$ it follows that either $R_{1}=-\infty$ or $R_{1}>-\infty$ and $y\left(R_{1}\right)=0$. Assuming now that $R_{1}>-\infty$, we may without loss of generality set $R_{1}=0$. Uniqueness of solutions of linear ODEs shows that $y_{1}=\frac{d}{d R} y(0)>0$, since we require that $r$ is nowhere vanishing on $I$. Expanding in a Taylor series about $R=0$ gives $y(R)=r^{3}(R)=y_{1} R+O\left(R^{2}\right)$, where the error term is controlled by $\|\tau\|_{\infty}+\|\xi\|_{\infty}$. Thus $\gamma=d R^{2}+\left(R^{2 / 3} y_{1}^{2 / 3}+O\left(R^{4 / 3}\right)\right)\left(d \theta^{2}+\sin ^{2} \theta d \phi^{2}\right)$ $=\left(\left(3 / y_{1}\right)^{2} r^{4}+O\left(r^{7}\right)\right) d r^{2}+r^{2}\left(d \theta^{2}+\sin ^{2} \theta d \phi^{2}\right)$, so the metric is singular at the symmetry centre $r=0$. Thus although the radial equation (9) is satisfied globally, the physical solution does not extend to points where $r=0$. This holds for $R_{2}$ as well as $R_{1}$.

Finally, since

$$
\theta_{-}=\mu-\frac{2}{r} \frac{d}{d R} r=-\frac{4}{r} \frac{d}{d R} r=-\frac{4}{3} \frac{d}{d R} \log y
$$


when $\theta_{+}=0$, the dynamical horizon condition (DH3) is equivalent to $\frac{d}{d R} r>0$ and $\frac{d}{d R} y>0$. Observe that (DH3) serves only to restrict the solution to the sub-interval $\left(R_{1}, R_{3}\right) \subset\left(R_{1}, R_{2}\right)$ on which $\frac{d}{d R} y>0$; without it the above solution could be extended beyond $R_{2}$, to regions where $\theta_{-} \geq 0$.

REMARK: The dynamical horizon metric behaviour $\gamma \sim c^{2} r^{4} d r^{2}+r^{2} d \Omega^{2}$ is similar to that of a constant mean curvature hypersurface near a point singularity [5]. However, (8) shows the spacetime curvature has components (ie. $G\left(e_{\perp}, \ell\right)$ ) which are unbounded as $r \rightarrow 0$ and we show in Corollary 6 that $r=0$ is a true spacetime curvature singularity.

\subsection{Examples}

As outlined above, the process of producing spherically symmetric initial data sets which satisfy the constraints and the dynamical horizon conditions from a given pair of freely chosen functions is very straightforward. We now illustrate this process with some examples.

Maximal Data with Co-Moving Matter: Setting $\xi=0$ corresponds to choosing the matter to be co-moving with the surface orthogonal observers, while setting $\tau=0$ results in the mean curvature of the initial data set vanishing, so the data is maximal. With these choices, equation (9) becomes simply $y^{\prime \prime}=0$, which has the general solution $y(R)=y_{1}\left(R-R_{0}\right)+y_{0}$. In this case, we may without loss of generality choose $R_{0}=0, y_{0}=0$, and $y_{1}=\alpha^{3}$ (for $\alpha>0$ ) so $y(R)=\alpha^{3} R$. It then follows that $r(R)=\alpha R^{\frac{1}{3}}$, and consequently the metric takes the form

$$
\gamma=d R^{2}+\alpha^{2} R^{\frac{2}{3}} d \Omega^{2}
$$

where $d \Omega^{2}$ indicates the round sphere metric. Calculating $\mu$ from (17) and substituting into the formula for $K$, we have

$$
K=\frac{2}{3 \alpha^{3} R} d R^{2}-\frac{1}{3 \alpha R^{\frac{1}{3}}} d \Omega^{2}
$$

Calculating $\rho$ from (8), we have $\rho(R)=\frac{1}{\alpha^{2} R^{\frac{2}{3}}}$. We note that for this example, the interval on which the solution is regular is $I=(0,+\infty)$. The solution is singular at $R=0$, where $r=0$. We also note that since $\frac{d y}{d R}=\alpha^{3}>0$, the entire solution satisfies condition (DH3). 
CMC Data with Co-Moving Matter: We again set $\xi=0$, but set $\tau$ equal to a non zero constant. The general solution ${ }^{4}$ to the equation for $y$ is $y(R)=\frac{y_{1}}{\tau}\left(1-e^{-R \tau}\right)+y_{0}$, which results in

$$
\gamma=d R^{2}+\left(\frac{y_{1}}{\tau}\left(1-e^{-\tau R}\right)+y_{0}\right)^{\frac{2}{3}} d \Omega^{2} .
$$

The expression for $K$ is a bit messy, but straightforward to obtain. The behavior of the function $y(R)$ for these examples depends on the sign of $\tau$. Recalling that $y_{0}$ and $y_{1}$ are both positive, we find that if $\tau$ is positive, then $y(R)$ has a zero for some negative value of $R$, and for large $R$ it approaches a constant. If $\tau$ is negative, then for negative $R$ the function $y$ either hits zero for some finite value $R_{1}$ or it approaches zero as $R \rightarrow-\infty$, while for large positive values of $R$ the function $y(R)$ is convex increasing and unbounded. Thus in these cases, either $I=(-\infty,+\infty)$ or $I=\left(R_{1},+\infty\right)$.

Since $R$ is a radial coordinate, which measures distance from the symmetry axis, to obtain a spherically symmetric dynamical horizon from this example, we need to restrict the interval $I$ to $(0,+\infty)$. When we do this, we find that while the metric is not degenerate as $R \rightarrow 0$, the curvature is unbounded as one approaches the axis.

Note that since $\frac{d y}{d R}=y_{1} e^{-R \tau}>0$ for any constant $\tau$, the (DH3) condition is satisfied for the full range of $I$.

Flat 3-metric: If we set $r(R)=R$, then $(\Sigma, \gamma) \simeq\left(\mathbb{R}^{3}, \delta\right)$. To see which expressions for $K$ and $\rho$ and $\xi$ are compatible with a flat metric $\gamma=\delta$, we substitute $y(R)=R^{3}$ into (9), giving

$$
6 R+3 \tau R^{2}-\frac{3}{2} \xi R^{3}=0 .
$$

Assuming co-moving matter $\xi=0$ leads to $\tau=-\frac{2}{R}$. From (7) we find that $\mu=-\frac{2}{R}$, so $K=-R d \Omega^{2}$ and the matter density is $\rho=\frac{1}{R^{2}}$. The data is clearly singular at $R=0$, and nowhere else. Without the co-moving assumption, we find that equation (9) implies that

$$
3 R\left(2+\tau R-\frac{1}{2} \xi R^{2}\right)=0 .
$$

Choosing, say, $\xi=\frac{1}{3 R^{2}}$, gives $\rho=\frac{2}{3 R^{2}}, \tau=-\frac{11}{6 R}$ and $\mu=-2 / R$ as before. One then readily constructs $K$, noting its singular behavior at the symmetry axis.

\footnotetext{
${ }^{4}$ In this case, since the solution is not linear in $R$, setting $y_{0}=0$ is a restriction. So we let $y_{0}$ and $y_{1}$ be any pair of positive constants.
} 
For all cases of this example, since $\frac{d y}{d R}=1>0$, condition (DH3) for a $\mathrm{SSDH}$ is satisfied everywhere.

\subsection{Physical Restrictions}

In the examples just discussed, we have made restrictions on the choice of the free data $\xi$ and $\tau$ based on mathematical convenience. In this section, we consider physically motivated restrictions, and some of their consequences.

Our first physical condition on a dynamical horizon $\Sigma$ is that there is at least one point on the symmetry axis, which we may normalise to $R=0$,

$$
r(R=0)=0 .
$$

Second, we recall that the definition of dynamical horizon requires that the foliation 2-surfaces $S_{R}^{2}$ should be contracting in the inward null direction $n=\ell_{-}$, but that this condition plays no direct role in the solution of the dynamical horizon equations (3) 9). Thus we consider explicitly the inner trapped condition

$$
\theta_{-}<0
$$

As shown by [9, 8, the inner trapped condition in spherical symmetry is non-evolutionary, meaning that if it is satisfied on a Cauchy surface then it holds throughout the globally hyperbolic development. We can also show that (18) follows from the outgoing momentum condition

$$
\xi \geq 0
$$

Finally, we recall that the conceptual picture of a dynamical horizon has it inside an event horizon, with the area function $r$ having a finite bound $r \leq 2 m(\infty)$ where $m(\infty)$ is the final Bondi mass. The examples above show that this condition is not satisfied by all solutions of the dynamical horizon constraint equations (5),(6). This motivates the black hole condition, that there is a constant $r^{*}>0$ with

$$
r^{*}:=\sup _{\left(0, R^{*}\right)} r
$$

where $R^{*}$ is maximal, in the following sense:

Definition 3 Given the data $\left(\tau, \xi, y_{1}, R^{*}\right) \in C^{0}(I) \times C^{0}(I) \times(0, \infty) \times(0, \infty]$ specified on the interval $I=\left(0, R^{*}\right)$, let the corresponding solution $(y, r, \mu, \tau, \rho, \xi)=$ : 
$\Sigma\left(\tau, \xi, y_{1}, R^{*}\right)$ be constructed as prescribed in Proposition 2 with $R_{0}=0$, $y_{0}=0$. If this solution satisfies $y>0$ and $\theta_{-}<0$ on $I$, then $\left(\tau, \xi, y_{1}, R^{*}\right)$ is called a $\mathrm{DH}$ data set. Such a DH data set is DH-extendible if there is a DH data set $\left(\hat{\tau}, \hat{\xi}, \hat{y}_{1}, \hat{R}\right)$ such that $\hat{R}>R^{*}$ and $\left.\hat{\tau}\right|_{(0, R)}=\tau,\left.\hat{\xi}\right|_{(0, R)}=\xi$ and $\hat{y}_{1}=y_{1}$. A DH data set $\left(\tau, \xi, y_{1}, R^{*}\right)$ is maximal if it is not DH-extendible. If the inner trapped condition is not assumed then we have a generalised $\mathrm{DH}$ data set, and a DH data set is weakly DH-extendible if it is extendible in the class of generalised DH data.

Proposition 4 Suppose $\Sigma\left(\tau, \xi, y_{1}, R^{*}\right)=(y, r, \mu, \tau, \rho, \xi)$ is a dynamical horizon constructed by Proposition 2 on $I=\left(0, R^{*}\right)$, so in particular, $\Sigma$ satisfies the axis condition (17).

(i) Suppose $\left(\tau, \xi, y_{1}, R^{*}\right)$ is a generalised DH data set, so the inner trapped condition (18) is not assumed a priori. If the outgoing momentum condition (19) is satisfied on a sub-interval $(0, \tilde{R})$ then the dynamical horizon is inner trapped on the same sub-interval, and $\lim _{R \uparrow \tilde{R}} \theta_{-}<0$.

(ii) Suppose the black hole (20) and inner trapped (18) conditions hold on $\left(0, R^{*}\right)$, where $R^{*}$ is maximal. Then $r(R)$ is increasing on $\left(0, R^{*}\right)$, $\lim _{R \uparrow R^{*}} \frac{d}{d R} r=0$, and either $R^{*}<\infty$ and $\theta_{-}\left(R^{*}\right)=0$, or $R^{*}=\infty$.

Proof: (i) It follows from $\theta_{+}=0$ and $n=\ell-2 e_{R}$ that $\theta_{-}=-4 \frac{d}{d R} \log r=$ $-\frac{4}{3} \frac{d}{d R} \log y$. Proposition 2 shows that if $y(0)=0$ then for small positive $R$ we have $\theta_{-} \simeq-\frac{4}{3} y^{\prime}(0) / R<0$. The master equation (91) and $\theta_{+}=0$ show that on $\Sigma$ we have

$$
\frac{d}{d R} \theta_{-}=\frac{3}{4} \theta_{-}^{2}-\tau \theta_{-}-2 \xi \leq \frac{3}{4} \theta_{-}^{2}-\tau \theta_{-},
$$

since $\xi \geq 0$ by assumption. Introducing $h=-1 / \theta_{-}$and integrating gives

$$
h(R) \leq e^{T(R)}\left(h(\epsilon)+\frac{3}{4} \int_{\epsilon}^{R} e^{-T(s)} d s\right),
$$

where $T(R)=\int_{\epsilon}^{R} \tau(s) d s$ and $\epsilon>0$ is chosen small enough that $h(\epsilon) \leq$ 1. Now $\tau \in C^{0}(\mathbb{R})$ so $h(R)$ is bounded above uniformly for finite $R$ and thus $\theta_{-}=-1 / h$ is negative as required; even more, $\theta_{-}$is locally uniformly negative, since $\int_{R}^{R+1} \tau(s) d s$ is bounded, for all $R$. 
(ii) The inner trapped condition $\theta_{-}<0$ and $\theta_{-}=-4 \frac{d}{d R} \log r$ shows that $\frac{d}{d R} r>0$. The master equation (9) and $\tau, \xi \in C^{0}(\mathbb{R})$ show $\frac{d}{d R} y=3 r^{2} \frac{d}{d R} r$ is bounded locally uniformly on $\mathbb{R}$, so $\lim _{R \uparrow R^{*}} \frac{d}{d R} r<\infty$ while $r$ is bounded away from 0 . Choosing any $C^{0}$ extension $(\tilde{\tau}, \tilde{\xi})$ of $(\tau, \xi)$ gives an $\tilde{R}>R^{*}$ and a generalised DH data set $\left(\tilde{\tau}, \tilde{\xi}, y_{1}, \tilde{R}\right)$ extending $\Sigma$, by continuity of $y$ at $R^{*}$. Continuity of $\frac{d}{d R} y$ implies that $\Sigma$ is DH-extendible, contradicting maximality of $R^{*}$, unless $\lim _{R \uparrow R^{*}} \frac{d}{d R} r=0$. Finally, if $R^{*}<\infty$ then continuity of $\frac{d}{d R} y$ shows that $\theta_{-}\left(R^{*}\right)=0$.

REMARK: Proposition 4 provides a natural picture of a dynamical horizon inside an event horizon, starting at the central axis and extending radialoutward and geodesically complete, with future endpoint at $i^{+}$. However, this picture relies on the assumptions we have made, in particular the inner trapped condition (18) and the continuity of the free fields $\tau, \xi$. Examples of marginally trapped tubes (cf. [2, 6]) show that the condition $\theta_{+}=0$ defines a hypersurface which may become null and timelike. In these examples, the dynamical horizon metric is inextendible as a spacelike hypersurface but not complete. The next results examine the solutions near the singular points 0 , $R^{*}$.

Lemma 5 Suppose $\Sigma=\Sigma\left(\tau, \xi, y_{1}, R^{*}\right)$ is a spherically symmetric dynamical horizon with $R^{*}<\infty$. Let $\left(E_{R}, E_{\perp}\right)$ be a frame along $\Sigma$ which is parallel transported by the spacetime connection. Then there is a finite boost (with parameter a) to the $\Sigma$-adapted frame $\left(e_{R}, e_{\perp}\right)$ at $R=R^{*}$,

$$
\begin{aligned}
& e_{R}=\frac{1}{2}\left(a-a^{-1}\right) E_{\perp}+\frac{1}{2}\left(a+a^{-1}\right) E_{R}, \\
& e_{\perp}=\frac{1}{2}\left(a+a^{-1}\right) E_{\perp}+\frac{1}{2}\left(a-a^{-1}\right) E_{R} .
\end{aligned}
$$

Furthermore, $\Sigma$ has spacelike radial unit tangent vector $e_{R}$ at $R=R^{*}$ and if $\lim _{R \uparrow R^{*}} \theta_{-}<0$ then $\Sigma$ is DH-extendible.

REMARK: The spacetime parallel transport provides a reference frame, uniformly equivalent to any other construction of spacetime frame, and therefore suitable for verifying whether or not $e_{R}$ goes null at $R^{*}$.

Proof: The extrinsic curvature $K_{a b}$ is defined by $K(X, Y)=g\left(X, \nabla_{Y} e_{\perp}\right)$, so $\nabla_{e_{R}} e_{R}=K\left(e_{R}, e_{R}\right) e_{\perp}$ and $\nabla_{e_{R}} e_{\perp}=K\left(e_{R}, e_{R}\right) e_{R}$. The parallel frame $\left(E_{R}, E_{\perp}\right)$ satisfies $\nabla_{e_{R}} E_{R}=\nabla_{e_{R}} E_{\perp}=0$. The corresponding null frames $\ell_{ \pm}=e_{\perp} \pm e_{R}$ and $L_{ \pm}=E_{\perp} \pm E_{R}$ are related by a boost with parameter 
$a=a(R)$, ie. $\ell_{ \pm}=a^{ \pm 1} L_{ \pm}$, and we find $\frac{d}{d R} \log a=K\left(e_{R}, e_{R}\right)=\tau-\mu=$ $\tau+2 \frac{d}{d R} \log r$. Integrating gives

$$
\frac{a(R)}{r(R)^{2}}=\frac{a\left(R_{1}\right)}{r\left(R_{1}\right)^{2}} \exp \left(\int_{R_{1}}^{R} \tau(s) d s\right),
$$

so the boost $a(R)$ is bounded while $r>0$ and $\int \tau<\infty$. Now $\theta_{-}\left(R^{*}\right)<0$ and $\theta_{-}=-4 \frac{d}{d R} \log r$ show that $\frac{d}{d R} r\left(R^{*}\right)>0$, so the dynamical horizon solution extends as before.

A similar argument can be used to show that the Einstein tensor is unbounded at the axis.

Corollary 6 Suppose $\Sigma$ is a spherically symmetric dynamical horizon in a spacetime $M$, which satisfies the axis condition (17) and $\int_{0}^{1} \tau(s) d s<\infty$. Then the central axis point $R=0, r=r(R=0)=0$ of $\Sigma$ lies on the singular set of the spacetime $M$.

Proof: Equation (8) shows that the component $G\left(e_{\perp}, \ell\right)$ is unbounded, and we must show this holds in all frames near $R=0$. It suffices to consider frames $L, N$ which are spacetime parallel along $\Sigma$. As in Lemma 5 the tangent frames $n, \ell$ and parallel frame $N, L$ are related by a boost with parameter $a=a(R)$; i.e., $\ell=a L$ and $n=a^{-1} N$, where $a=r^{2} O(1)$ as $R \rightarrow 0$ by (22). It follows from $r^{-2}=\frac{1}{2}(G(\ell, n)+G(\ell, \ell))=\frac{1}{2}\left(G(L, N)+a^{2} G(L, L)\right)$ that $G(L, L)=O\left(r^{-6}\right)$ is the required unbounded coefficient. Note also that the boost $n=O\left(r^{-2}\right) N$ so $\Sigma$ is tangent to the past null cone at the central axis point; see ([], Figure 3.).

\section{Spacetime Picture}

Let $(M, g, T)$ be a spherically symmetric spacetime which satisfies the Einstein equations $G_{\mu \nu}=8 \pi \mathrm{G} T_{\mu \nu}$ with stress-energy $T_{\mu \nu}$, and consider the question of finding a dynamical horizon in $M$. If one were to exist in $(M, g, T)$, then the initial data on this dynamical horizon would necessarily satisfy the conditions discussed in Section [3] including (8). Since (8) depends on the adapted frame $\left(e_{\perp}, e_{R}\right)$, it is not easy to check directly from the spacetime fields. One can however state an equivalent slice-independent condition: 
Lemma 7 Let $W \subseteq M$ be a spacetime region with stress-energy tensor $T$ satisfying the strict null energy condition (SNEC)

$$
T(L, L)>0 \text { for all radial null vectors } L \text { in } W \text {. }
$$

Then the following are equivalent:

(i) There is a future null frame $(\ell, n)$ with $g(\ell, n)=-2$ satisfying the condition

$$
\frac{1}{2} r^{2} G(n, \ell)=4 \pi \mathrm{G} r^{2} T(n, \ell)<1 .
$$

(ii) There is a spacetime frame $\left(E_{\perp}, E_{R}\right)$ satisfying (8).

REMARK: The Lorentz-invariant condition (24) arises frequently: for example, in [6] the sign of the $C$-function ([6] equation (2.3))

$$
C=\frac{\frac{1}{2} r^{2} G(\ell, \ell)}{1-\frac{1}{2} r^{2} G(\ell, n)}
$$

(or more accurately, the sign of $C^{-1}$ ) determines the causal character (spacelike/null/timelike) of the marginally trapped tube (MTT). Another example is the evolution equation [9]

$$
\frac{\partial^{2}\left(r^{2}\right)}{\partial u \partial v}=-\Omega^{2}\left(1-\frac{1}{2} G(\ell, n) r^{2}\right)
$$

for the areal function $r^{2}(u, v)$ in double null coordinates $d s^{2}=-\Omega^{2}(u, v) d u d v+$ $r^{2}\left(d \theta^{2} \sin ^{2}(\theta) d \phi^{2}\right)$.

Proof: To show that $(i) \Rightarrow(i i)$, we check the effect of a Lorentz boost on $T\left(e_{\perp}, \ell\right)$. The boosted frame $L=a \ell, N=a^{-1} n, E_{\perp}=\frac{1}{2}(L+N)=$ $\frac{1}{2}\left(a \ell+a^{-1} n\right)$ satisfies

$$
2 T\left(E_{\perp}, L\right)=T(N, L)+a^{2} T(L, L) ;
$$

hence it follows from the SNEC (23) and the Lorentz invariance of (24) that there is a unique $a>0$ such that $T\left(E_{\perp}, L\right)=\frac{1}{2}\left(4 \pi \mathrm{G} r^{2}\right)^{-1}$, which is equivalent to the condition (8). The converse $(i i) \Rightarrow(i)$ follows from the above boost relation, together with the definitions of $\rho$ and $\xi$ in terms of $T$.

It follows from this Lemma that, if we are given a spacetime which satisfies the SNEC, then inequality (24) is a necessary condition for it to contain a 
dynamical horizon. We now look to find sufficient conditions for existence. Suppose that in our given spacetime $(M, g, T)$ there is a non-empty region $W \subseteq M$ in which the SNEC condition and (24) both hold. From the lemma, it follows that through every point in $W$ there is a spacelike vector $e_{R}$ such that the adapted frame $\left(e_{\perp}, e_{R}\right)$ satisfies (8). The integral curves of the vector field $e_{R}$ give a foliation of $W$ by spacelike hypersurfaces which satisfy (8). It follows from the results of Section 3 that if the spacetime contains a dynamical horizon, it must coincide with a leaf of this foliation.

To see that condition (8), whilst necessary for a given leaf to be a dynamical horizon, is not sufficient ${ }^{5}$, we use the constraint equations with (8) and the expression $\theta_{+}=\mu+\frac{2}{r} \frac{d}{d R} r$ for the future outer expansion $\theta_{+}$to derive a differential equation for $\theta_{+}$. Specifically, writing (6) in terms of $\theta_{+}$and eliminating $\frac{d^{2} r}{d R^{2}}$ with (15), we obtain

$$
\frac{d}{d R} \theta_{+}+\left(\frac{3}{4} \theta_{+}-\tau\right) \theta_{+}=\frac{1}{r^{2}}-\rho-\xi
$$

Clearly if we choose a spacelike hypersurface with adapted frame $\left\{e_{\perp}, e_{R}\right\}$ relative to which $\rho+\xi=\frac{1}{r^{2}}$ (which Lemma 2 guarantees that we always can do) then the right hand side of (27) vanishes, and we see that (27) admits the solution $\theta_{+}=0$. It then follows from ODE uniqueness that the sign of $\theta_{+}$is fixed on each leaf. Hence if $p \in W$ and $\theta_{+}(p)=0$ then the leaf through $p$ is a dynamical horizon. Finally, it follows from the Raychaudhuri equation

$$
D_{\ell} \theta_{+}=-\frac{1}{2} \theta_{+}^{2}-8 \pi \mathrm{G} T(\ell, \ell)
$$

and from the strict null energy condition that $D_{\ell} \theta_{+}<0$. Presuming the spacetime to be smooth, we find that there are four possibilities:

(a) The region $W$ has $\theta_{+}>0$ everywhere, so that it is entirely untrapped, and there is no dynamical horizon.

(b) The region $W$ has $\theta_{+}<0$ everywhere, so that it is entirely trapped, and there is no dynamical horizon.

\footnotetext{
${ }^{5}$ We have shown in Section 3 that while (8) holds, for any choice of a pair of free functions, we can construct initial data so that the hypersurface with that data is a dynamical horizon for the spacetime development of that data. It does not, however, follow that any initial data set satisfying (8) is a dynamical horizon: we show here that the additional condition $\theta_{+}(p)=0$ for at least one point is necessary.
} 
(c) The region $W$ contains a single leaf with $\theta_{+}=0$, but $\theta_{-} \geq 0$ everywhere on this leaf. So there is no dynamical horizon.

(d) The region $W$ contains a single leaf with $\theta_{+}=0$, and on some (possibly proper) subset of this leaf, $\theta_{-}<0$. This subset is a dynamical horizon.

In summary, we have shown the following:

Proposition 8 Let $(M, g, T)$ be a smooth spacetime which satisfies the strict null energy condition (23).

1. If condition (24) is satisfied nowhere in $M$, then the spacetime contains no dynamical horizons.

2. If there exists a non-empty connected region $W \subseteq M$ in which condition (24) holds, and if the outward null expansion function $\theta_{+}$has constant sign (with no zeroes) then $W$ contains no dynamical horizon and is everywhere trapping or non-trapping.

3. If there exists a non-empty connected region $W \subseteq M$ in which condition (24) holds, and if the expansion $\theta_{+}$takes on both positive and negative values in $W$, then $W$ contains a unique spacelike hypersurface with $\theta_{+}=0$. The data on this hypersurface satisfy (8)), and it is (entirely or partly) a dynamical horizon if and only if $\theta_{-}<0$ on all of it or part of it. In addition, this hypersurface divides $W$ into a trapping region to the future and a non-trapping region to the past.

REMARK: It is worth noting that, unlike the situation for dynamical horizons in non-spherical spacetimes, the spherically symmetric construction given here does not depend on any choice of (spherically symmetric) time slicing. This leads to a much stronger uniqueness statement (Proposition 8) than is available in the general case.

\section{Conclusion}

While the set of spacetimes which are spherically symmetric is very special, it is clear from physical considerations that, so long as matter is present, dynamical horizons can form in them. We determine here the necessary conditions for spherically symmetric spacetime to contain a dynamical horizon, and sufficient conditions as well. We also demonstrate their uniqueness, and 
show how to systematically construct initial data sets which serve as dynamical horizons for their spacetime developments (with two free functions parametrizing the collection of all such data sets).

The situation regarding dynamical horizons in non-spherically symmetric spacetimes, is of course considerably more complicated. Still, a full understanding of the spherically symmetric case provides a good first step toward uncovering the properties of dynamical horizons more generally.

\section{Acknowledgments}

We thank Abhay Ashtekar, Greg Galloway and Dan Pollack for useful discussions, and the Isaac Newton Institute and the Australian National University for hospitality at various stages. The work of JI is partially supported by NSF grant PHY-0354659 at Oregon, and RB acknowledges support from the Clay Institute and the Australian Research Council.

\section{References}

[1] L. Andersson, M. Mars and W. Simon, Local existence of dynamical and trapping horizons, gr-qc/0506013.

[2] A. Ashtekar and G. Galloway, Some Uniqueness results for dynamical horizons, gr-qc/0503109.

[3] A. Ashtekar and B. Krishnan, Dynamical horizons: energy, angular momentum, fluxes and balance laws, Phys. Rev. Lett. 89 261101, 2002.

[4] A. Ashtekar and B. Krishnan, Isolated and dynamical horizons and their applications, Liv. Rev. Rel. 10, 2004.

[5] R. Bartnik, Isolated singular points of Lorentzian mean curvature hypersurfaces., Indiana Math. J., 38 811-827, 1989.

[6] I. Booth, L. Brits, J. Gonzalez, and C. Van Den, Broeck, Marginally trapped tubes and dynamical horizons, gr-qc/0506119.

[7] Y. Choquet-Bruhat and R. Geroch, Global aspects of the Cauchy problem in general relativity, Comm. Math. Phys., 14 329-335, 1969. 
[8] D. Christodoulou, Self-gravitating relativistic fluids: a two-phase model, Arch. Rat. Mech. Anal. 130 343-400, 1995.

[9] M. Dafermos, Spherically symmetric spacetimes with a trapped surface, Class. Quantum Grav. 22 2221-2232, 2005.

[10] Y. Foures-Bruhat, Théorème d'existence pour certains systèmes d'équations aux dérivées partielles non-linéaires, Acta Math., 88 141225, 1952.

[11] R. Wald, Gravitation, Chicago U. Press, 1984. 